\title{
Synthesis and optimization of immunomodulating hydrogel for biomedical application
}

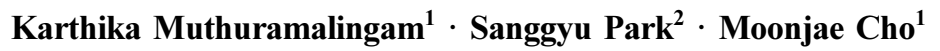

Received: 22 October 2018 / Accepted: 28 October 2018 / Published Online: 31 December 2018

(C) The Korean Society for Applied Biological Chemistry 2018

\begin{abstract}
Treatment towards wound healing, a complex and dynamic process, has been given a great deal of efforts in the last few decades. Focus has been imposed on developing wound dressings that meet the requirements for proper wound healing. In this study, hydrogel made from blends of poly (vinyl alcohol) and $\beta$-1,6-branched- $\beta$-1,3-glucan (beta-glucan) were synthesized by modified solvent casting method for wound dressing application. Optimization of hydrogel composition and analysis of wound dressing parameters such as stability and fluid uptake capacity (in the presence of water, saline and different $\mathrm{pH}$ solutions) has been studied. The result indicated that the PVA/beta-glucan hydrogel hold its structural integrity even at alkaline $\mathrm{pH}(\mathrm{pH} \sim 9)$ and upholds fluids four times of its original weight. Thus, the developed hydrogel is expected to be a promising candidate as wound dressing.
\end{abstract}

Keywords $\beta$-1,6-branched- $\beta$-1,3-glucan · Hydrogel · Poly (vinyl alcohol) $\cdot$ Solvent-casting $\cdot$ Wound dressing

\section{Introduction}

Being the first line of defence, skin forms the protective barrier of the human body with the dense surface and corneous layer.

Moonjae Cho( $₫)$

E-mail: moonjcho@jejunu.ac.kr

${ }^{1}$ Department of Biochemistry, School of Medicine, Jeju National University, Jeju 63241, Republic of Korea

${ }^{2}$ Division of Life \& Environmental Science, Deagu University 712-714, Republic of Korea

This is an Open Access article distributed under the terms of the Creative Commons Attribution Non-Commercial License (http://creativecommons. org/licenses/by-nc/3.0/) which permits unrestricted non-commercial use, distribution, and reproduction in any medium, provided the original work is properly cited.
Healing process of the skin wounds is a complex process, aiming to restore several functional damages experienced by the wounded skin $[1,2]$. From a long time, use of cotton, gauze etc. is used in the treatment of wound healing [3]. However, the resulting dry wound bed leads to the formation of scab thereby extending the healing time through retarding the rate of epithelialization [4]. It was when Winter [5] demonstrated that moist wound bed accelerates the wound healing mechanism, research has been focused on exploring biomaterials that provides moisture to the wound bed along with playing the role of physiological barrier and shield which protects the wound as well as aids in the removal of exudates oozing from the wound site.

Hydrogel are three dimensional polymeric networks that can absorb and retain huge amount of water inside it, when kept in aqueous medium [6]. They are made from homo-/co-polymeric grids synthesized from synthetic and/or natural polymers. Due to its tendency to absorb water, they form soft consistency when swell and thereby closely mimics the functional tissue and cellular environment [7]. Hydrogel made from natural biopolymers such as collagen, cellulose, xanthan, alginate, hyaluronan, carrageenans etc. and partial synthetic/synthetic polymers such as poly (vinyl alcohol), poly (ethylene glycol), poly vinyl pyrollidone, polyurethane etc are widely investigated for their application in drug delivery, wound dressings, tissue engineering etc [8,9]. Significant properties such as water holding ability, hydrophilicity, biocompatibility, biodegradability, non-cytotoxicity and non-irritability make the hydrogel one of the promising candidate to be used as wound dressings [10].

PVA is a water soluble- and non-carcinogenic polymer extensively studied in the medical industries in the form of film, fibre, powder, thin coatings, capsule coverings etc [11-13]. Hydrogels made from PVA has been given particular interest in the field of wound dressings due to its transparency, impermeable barrier to bacteria, permeability to small molecules etc. Inspite of these substantial properties, PVA based hydrogel suffer from insufficient elasticity and stiffer membrane with imperfect hydrophilicity [14]. Blending PVA with other polymers enhances the setbacks of PVA 
hydrogel thereby improving the wound healing process. $\beta-1,6-$ branched- $\beta$-1,3-glucan (Beta-glucan), a complex hetero-polysaccharide, is a potent activator of macrophage cells along with inducing the proliferation of dermal cells [15].

In this study, hydrogel made from blends of PVA and betaglucan is synthesized through modified solvent casting method and optimization of the composition of the hydrogel is analysed for desirable characteristics. Using the blends of PVA and betaglucan, it is expected that the resulting hydrogel can act as a promising wound dressing; wherein the fungal polysaccharide accelerates wound healing and mechanical stability is provided by poly vinyl alcohol.

\section{Materials and Methods}

\section{Materials}

Poly vinyl alcohol (99+\% hydrolyzed) and Glutaraldehyde (25\% in $\mathrm{H}_{2} \mathrm{O}$, Grade 1) was purchased from Sigma-Aldrich. Betaglucan (average MW, $3.5 \times 10^{6} \mathrm{Da}$ ) was provided by Quegen Biotech Co., Ltd. Glycerol ( $>99.0 \%$ purity, MW 92.09) was purchased from Biosesang Inc, Gyeonggi-do, Korea. All the chemicals were used without further purification.

\section{Optimization of hydrogel}

The hydrogel was synthesized through modified solvent casting method. Hydrogel formulation with different composition of the initial precursors were made and allowed to air dry for 48-72 hours after pouring onto petri dish.

Briefly, the initial precursor molecules, poly (vinyl alcohol) and beta-glucan were mixed, according to the composition given in Table 1, under magnetic stirring for $10 \mathrm{~min}$ at $60^{\circ} \mathrm{C}$, such that the final concentration of PVA and beta-glucan in the final solution is $2 \%(\mathrm{w} / \mathrm{v})$ and $0.2 \%(\mathrm{w} / \mathrm{v})$ respectively. Glycerol and Glutaraldehyde were added to the solution sets as per the volume indicated in the above-mentioned table and stirring was continued for another 30 min in-order to achieve homogenous solution. The solution was then poured onto petri dish and was allowed to air dry for a period of 2-3 days, later which it can be peeled off evenly as a hydrogel film. The colour of the film was noted down after peeling the film from the petri dish. The weight of the hydrogel per $5 \mathrm{~mL}$ of the initial solution were noted down after $48 \mathrm{~h}$ of air drying. With the use of tissue paper, the film was blotted and the water absorbed in the tissue paper was classified as high, medium and low upon comparing with each other.

\section{Stability of the hydrogel}

The hydrogels were cut into $1 \mathrm{~cm} \times 1 \mathrm{~cm}$ and were immersed in water for $24 \mathrm{~h}$ at $37^{\circ} \mathrm{C}$. At the end of $24 \mathrm{~h}$, the samples were taken out from the medium and were blotted using tissue paper. The ability of the hydrogel to remain in its stable form was noted down.

\section{Fluid uptake capacity}

The stable hydrogel with PVA/Beta glucan formulation was taken for fluid uptake study. Briefly, $1 \mathrm{~cm} \times 1 \mathrm{~cm}$ was cut from the hydrogel film and immersed in solution (distilled water and $0.9 \%$ $\mathrm{NaCl})$ at $37^{\circ} \mathrm{C}$. At particular intervals of time, the film was taken out from the immersion medium and the surface water was gently blotted using tissue paper. The weight of the sample before and after immersion in immersion medium was noted. The swelling ratio was calculated using the following formulae:

$$
\text { Swelling ratio }(\%)=\left(\mathrm{W}_{\mathrm{t}} / \mathrm{W}_{\mathrm{i}}\right) * 100
$$

where, $\mathrm{W}_{\mathrm{i}}$ and $\mathrm{W}_{\mathrm{t}}$ are the weights of the hydrogel sample before immersion and at particular intervals of time after immersion respectively.

\section{Effect of pH on fluid uptake capacity}

The effect of $\mathrm{pH}$ on swelling ratio of the hydrogel samples were observed at five different $\mathrm{pH}$ conditions ( $\mathrm{pH} \mathrm{4,} \mathrm{5.8,} \mathrm{7,} \mathrm{7.4,} \mathrm{and} \mathrm{9).}$ Briefly, the hydrogel samples were weighed and immersed in different $\mathrm{pH}$ solution at $37^{\circ} \mathrm{C}$. At specific time intervals, these samples were taken out from the immersion medium and after removing the surface water, the weights were again noted down. The effect of $\mathrm{pH}$ on swelling ratio was calculated using the formulae given in equation (1).

\section{Results and Discussion}

The prepared hydrogel solutions (from Table 1) poured onto the petri-dish forms hydrogel when left to atmospheric air drying for 48-72 h. The test groups to which glutaraldehyde solution was added, showed switch in colour from transparent solution to translucent yellow shades, marking the crosslinking process (between functional groups of poly (vinyl alcohol) and betaglucan molecules) taking place in the hydrogel formation step. The remaining test groups in which glutaraldehyde is absent, remained transparent even after the formation of hydrogel. Also, addition of $\beta$-glucan and Glycerol to the hydrogel precursor solution, increases the weight of the hydrogel (measured per $5 \mathrm{~mL}$ of the prepared solution after 48-72 $\mathrm{h}$ of air drying during the hydrogel formation process).

In addition, all the test groups containing beta-glucan showed high surface moisture while the rest of the test groups exhibited no moisture content (showed in Table 2). In order to test the stability of the formed hydrogel, $1 \mathrm{~cm} \times 1 \mathrm{~cm}$ sample from each test group were immersed in water solution for $24 \mathrm{~h}$ and were looked for their ability to hold the structure, even after uptake of the fluid. It was found out that the test groups 2 and 7 exhibited good stability apart from the hydrogel made of PVA alone (either with or without glutaraldehyde and glycerol).

The starter materials used for hydrogel synthesis were acidic (beta-glucan [pH 3.5 to 4.0] and PVA [pH 5.6 to 5.8]), making the 
Table 1. Optimization parameters in the synthesis of hydrogel and the corresponding weight of the formed membrane obtained per mL of the hydrogel solution kept at air drying for $48 \sim 72 \mathrm{~h}$

\begin{tabular}{|c|c|c|c|c|c|c|c|c|}
\hline Test group & 1 & 2 & 3 & 4 & 5 & 6 & 7 & 8 \\
\hline Beta Glucan (w/v) & - & 0.004 & - & 0.004 & - & 0.004 & 0.004 & - \\
\hline $\operatorname{PVA}(w / v)$ & 0.04 & 0.04 & 0.04 & 0.04 & 0.04 & 0.04 & 0.04 & 0.04 \\
\hline Glycerol (v/v) & 0.04 & 0.04 & 0.04 & 0.04 & - & - & 0.04 & 0.04 \\
\hline $1 \%$ GA (v/v) & 0.04 & 0.04 & - & - & - & - & 0.1 & 0.1 \\
\hline $\begin{array}{l}\text { Weight }(\mathrm{g}) \text { of hydrogel (per } 5 \mathrm{~mL} \text { ) } \\
\text { after } 48 \sim 72 \mathrm{~h} \text { of atmospheric drying }\end{array}$ & 1.1438 & 1.3034 & 0.7735 & 1.2493 & 0.2172 & 0.7211 & 1.2632 & 0.4975 \\
\hline
\end{tabular}

Table 2 Physical characteristics of the as-prepared hydrogel

\begin{tabular}{ccc}
\hline \hline Set & Colour & Surface water \\
\hline 1 & Yellow transparent & Lowest \\
2 & Yellow transparent & High \\
3 & Colorless transparent & Medium \\
4 & Colorless transparent & High \\
5 & Colorless transparent & No \\
6 & Colorless transparent & High \\
7 & Yellow transparent & High \\
8 & Yellow transparent & Lowest \\
\hline Inference & Addition of GA leads to & Adding $\beta$-Glucan \& Glycerol \\
& transparent yellow colour & gives moist nature to the gel
\end{tabular}

synthesized hydrogel also acidic. Thus, the milieu provided by this acidic hydrogel is expected to favour the activation and survival of neutrophils and migration of epidermal cells to the wound site, which in turn helps in intensifying the pace of wound healing [16]. Apart from the soluble beta-glucan, glycerol, a trihydroxy alcohol, was added to synthesize the hydrogel. Glycerol helps to improve skin hydration and epidermal barrier repair during the complicated multistep wound healing process [17]. In addition, the stiffness imparted by the PVA to the hydrogel was lessened with the addition of glycerol, in addition to the effect of beta-glucan.

It was reported earlier that higher the glucan content, the less

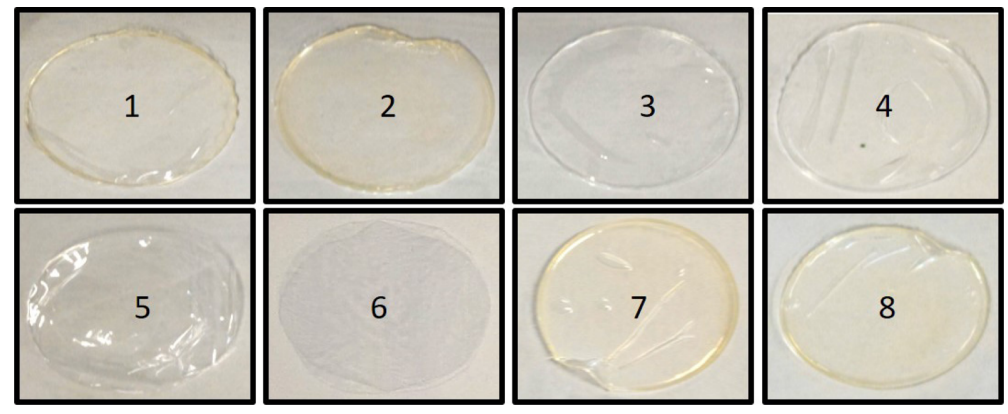

Fig. 1 Visual observation of the different hydrogel groups (prepared as mentioned in Table 1)

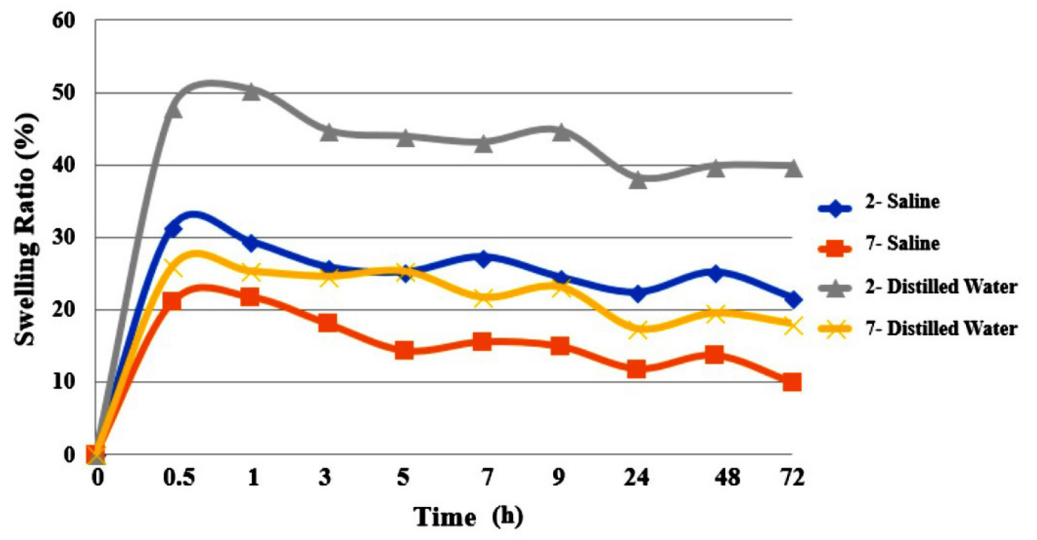

Fig. 2 Fluid Uptake Capacity of the hydrogel (Test group 2 and 7 given in Table 1). Briefly $1 \mathrm{~cm} \times 1 \mathrm{~cm}$ hydrogels were immersed in solutions (distilled water and saline $(0.9 \% \mathrm{NaCl}))$ and their corresponding weights before and after immersion at particular time intervals were measured for calculating the swelling ratio of the test samples 


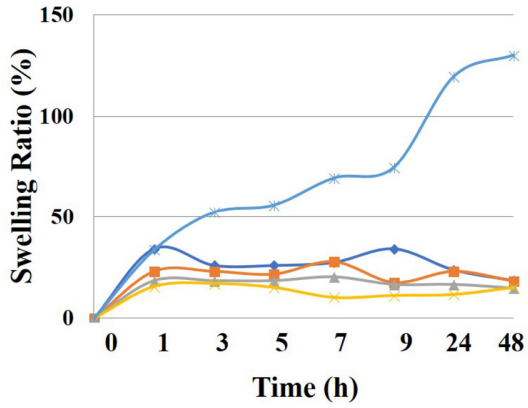

Test Group : 2

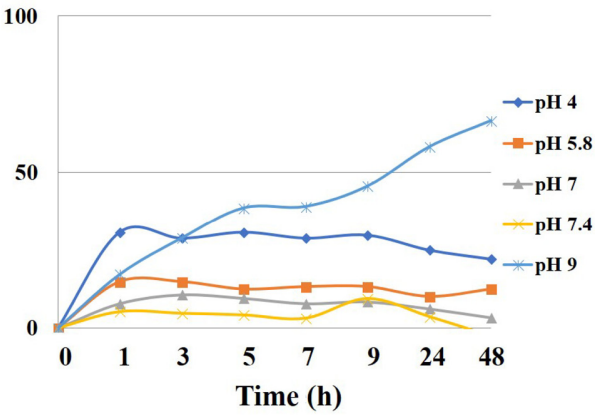

Test Group : 7

Fig. 3 Effect of $\mathrm{pH}$ on fluid uptake capacity of the hydrogel (Test group 2 and 7 given in Table 1)

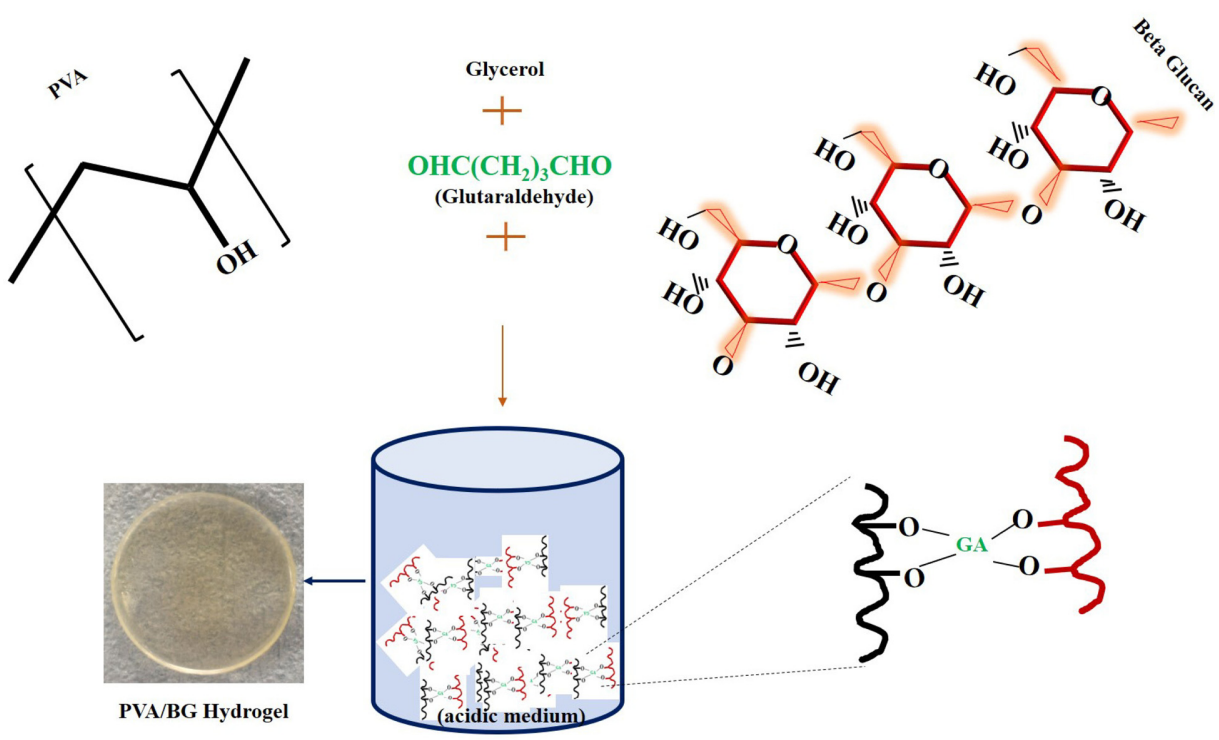

Fig. 4 Schematic representation of the synthesis of PVA/beta-glucan hydrogel and the possible mechanism of hydrogel formation

wound healing in the inflammatory phase; whereas higher the PVA content, the stiffer and moisture-less the hydrogel was, making it unsuitable for wound dressing application [14]. The blending ratio of the polymers used in this study (polyvinyl alcohol and beta-glucan) were carefully selected so that the effects of the hydrogel on wound healing, as well as its mechanical stability in body fluids, was greatly enhanced. Thus, the hydrogel (test groups 2 and 7) with appropriate polymer blends and composition with suitable moisture and flexible nature were selected for further analysis of the fluid uptake capacity, a desirable parameter in wound dressing application.

Swelling ratio acts as an index for analysing the fluid uptake capacity of the hydrogel. Solutions such as de-ionized water, saline solution and solutions with different $\mathrm{pH}$ were used for studying the effect of solutions on water uptake ability of the hydrogel. As the former two solutions are used to clean wounds and the saline solution resembles body fluid, these solutions were selected for the swelling study. In addition, normal human skin is slightly acidic to prevent microbial invasions. However, some medical conditions may alter the skin; therefore, the swelling behaviour of the hydrogel was studied using solutions with solutions with different $\mathrm{pH}$ values (4.0, 5.8, 7, 7.4, and 9.0). Thus, solutions with different $\mathrm{pH}$ conditions were involved in analysing the fluid uptake capacity of the hydrogels. Briefly, the hydrogel was cut and immersed into different solutions. At the required time intervals, the immersed hydrogel pieces were removed from each solution and, with blotting paper, the solution adsorbed onto the hydrogel surface was carefully removed. The weight of the individual pieces was determined before and after immersion, and the swelling ratio was calculated. The water holding capacity of the hydrogel (Test group: 7) comparable to its dry weight was found to be $93.22 \%$ (data not shown).

It was found out that the test group ' 2 ' surpasses the water uptake capacity upon comparing the test group '7', when immersed in all the three types of solution. Also, the water uptake capacity of the hydrogel is higher for both the groups upon immersing in distilled water when comparing the saline solution. Infected wound beds and exudate fluid have $\mathrm{pHs}$ ranging from 7.5 to 8.9. 
Hence, it is important for the hydrogel to hold its structural integrity together with removing the exudate fluids from the alkaline wound bed. When testing the effect of $\mathrm{pH}$ on swelling ratio of the hydrogel, the hydrogel immersed in a solution with an alkaline $\mathrm{pH}$ showed drastic swelling of more than four times its original weight followed by water uptake capacity at $\mathrm{pH} 4.0$ and 7.4. At $\mathrm{pH} 5.8$ and a neutral state, the swelling capacity of the hydrogel was reduced. In all the $\mathrm{pH}$ conditions, the hydrogel remained stable. In addition, the as-prepared membrane (Test group: 7) was subjected to drying by keeping it in hot air oven for a period of 7 days, followed by analysing the swelling capacity of the dried membrane. It was found out that the dried membrane still holds its capacity to swell as comparable to the swelling ratio exhibited by non-heat-treated membrane (Fig. 4, Supplementary Fig. 1).

Acknowledgment This research was supported by the 2018 scientific promotion program funded by Jeju National University.

\section{References}

1. Chen X, Zhang M, Wang X, Chen Y, Yan Y, Zhang L, Zhang L (2017) Peptide-modified chitosan hydrogels promote skin wound healing by enhancing wound angiogenesis and inhibiting inflammation. Am J Transl Res 9: 2352-2362

2. Devalliere J, Dooley K, Yu Y, Kelangi SS, Uygun BE, Yarmush ML (2017) Co-delivery of a growth factor and a tissue-protective molecule using elastin biopolymers accelerates wound healing in diabetic mice. Biomaterials 141: 149-160

3. Pandiyarasan V, Archana J, Pavithra A, Ashwin V, Navaneethan M, Hayakawa Y, Ikeda H (2017) Hydrothermal growth of reduced graphene oxide on cotton fabric for enhanced ultraviolet protection applications. Materials Letters 188: 123-126

4. Kamoun EA, Kenawy ERS, Chen X (2017) A review on polymeric hydrogel membranes for wound dressing applications: PVA-based hydrogel dressings. J Adv Res 8: 217-233
5. Jones J (2005) Winter's concept of moist wound healing: a review of the evidence and impact on clinical practice. J Wound Care 14: 273-276

6. Madaghiele M, Sannino A, Ambrosio L, Demitri C (2014) Polymeric hydrogels for burn wound care: Advanced skin wound dressings and regenerative templates. Burns Trauma 2: 153-161

7. Gong, CY, Wu QJ, Wang YJ, Zhang DD, Luo F, Zhao X, Wei YQ, Qian ZY (2013) A biodegradable hydrogel system containing curcumin encapsulated in micelles for cutaneous wound healing. Biomaterials 34 : $6377-6387$

8. Mogo ${ }^{\circ}$ anu GD, Grumezescu AM (2014) Natural and synthetic polymers for wounds and burns dressing. Int J Pharm 463: 127-136

9. Caló E, Khutoryanskiy V V (2015) Biomedical applications of hydrogels: A review of patents and commercial products. European Polymer Journal 65: 252-267

10. Murakami K, Aoki H, Nakamura S, Nakamura S, Takikawa M, Hanzawa M, Kishimoto S, Hattori H, Tanaka Y, Kiyosawa T, Sato Y, Ishihara M (2010) Hydrogel blends of chitin/chitosan, fucoidan and alginate as healing-impaired wound dressings. Biomaterials 31: 83-90

11. Kamoun EA, Chen X, Mohy Eldin MS, Kenawy ERS (2015) Crosslinked poly(vinyl alcohol) hydrogels for wound dressing applications: A review of remarkably blended polymers. Arabian Journal of Chemistry $8: 1-14$

12. Venugopal G, George R, Raghavan N, Srinivas T, Dakshinamurthy A, Paul AJ, Marahatta AB (2015) Structural and Mechanical Properties of MgO-Poly(Vinyl Alcohol) Nanocomposite Film. Advanced Science, Engineering and Medicine 7: 457-464

13. Thermal I, Nanocomposites MU, Jeyasubramanian K, Muthusankar E, Hikku GS, Selvakumar N, Thermal I, Behav FR (2018) US Accepted manuscript to appear in IJN CR. doi:10.1142/S0219581X18500254

14. Huang MH, Yang MC (2008) Evaluation of glucan/poly(vinyl alcohol) blend wound dressing using rat models. International Journal of Pharmaceutics 346: 38-46

15. Grip J, Engstad RE, Skjæveland I, Škalko-Basnet N, Holsæter AM (2017) Sprayable Carbopol hydrogel with soluble beta-1,3/1,6-glucan as an active ingredient for wound healing-Development and in-vivo evaluation. Eur J Pharm Sci 107: 24-31

16. Barker H, Aaltonen M, Pan P, Vähätupa M, Kaipiainen P, May U, Prince S, Uusitalo-Järvinen H, Waheed A, Pastoreková S, Sly WS, Parkkila S, Järvinen TA (2017) Role of carbonic anhydrases in skin wound healing. Exp Mol Med 49: e334

17. Fluhr JW, Darlenski R, Surber C (2008) Glycerol and the skin: Holistic approach to its origin and functions. Br J Dermatol159: 23-34 